Chapter 2

\title{
Virtual Reality in Medicine - Going Beyond the Limits
}

\author{
Florin Graur \\ Additional information is available at the end of the chapter \\ http://dx.doi.org/10.5772/59277
}

\section{Introduction}

In the last years technology had an explosive evolution in the development of new minimal invasive medical instruments and led to a novel need and a brand new field: virtual medicine.

Virtual reality is an "in silico" simulation of a real situation. In particular, in surgery, dimensional modeling of deformable objects with spatial characteristics and specific elastic-dynamic behavior is required. In addition, simulation of tools and their action against the organs in the virtual environment is also required.

The purpose of virtual medicine is to minimize the direct exploration of the human body in order to provide a virtual model for those who want to learn new techniques and to provide advanced treatment options for those who perform delicate maneuvers or in areas inaccessible by conventional methods: in short to minimize the invasiveness of treatment.

Also a number of virtual techniques tend to be held in the virtual environment of the Internet, allowing remote accessibility and an increased number of users.

This chapter is a literature review that explores the current virtual techniques used in research, education, treatment and development trends of these methods in the future.

\section{Short history}

First virtual system proposed in medicine was developed by Robert Mann in 1965 [1]. It was used to decide what the best procedure for a orthopedic disease would be. Also this system was used for training residents [2].

In the 60s, the first simulators with 3D images appeared. In the 80s the head mounted display (HDM) were developed and the term "virtual reality" was defined. Over a decade later came 
the application of virtual reality used in medical education (simulator of colonoscopy and upper gastrointestinal endoscopy) and the first simulator for laparoscopy appeared. The simulation systems became more advanced in time, but it was necessary to develop tactile interaction between man and machine. Haptic systems involve the transmission of information from human to computer and vice versa. Virtual reality nowadays is closely linked to haptic devices with force-feedback [2].

\section{Research methods using virtualization in medicine}

Among research methods using virtual medicine there are a number of examples of software that is used for the three-dimensional reconstruction of protein molecules. Understanding how proteins (whose structure of amino acids is known) are "packed" to reach the three-dimensional structure of the molecule and especially understanding errors of "packing", helps researchers in deciphering the molecular biology of diseases such as: Alzheimer's disease, mad cow disease, Huntington's disease, Parkinson's disease, cystic fibrosis and cancers.

The need for high computing power has led to the creation of online communities such as Rosetta@Home, Human Proteome Folding and Folding@Home. Within these communities an internet user can offer their unused computing capacity of its personal computer to participate in these projects [3-5].

The usefulness of these programs is to understand the physico-chemical properties of different types of proteins and different types of interactions between amino-acids, as well as the evaluation of local energy of short sequences of amino acids. This way the researchers could identify the structures with the lowest energy (i.e., the greatest stability) resulting in the most stable structure of the native protein. Moreover, one can understand the most predictable possibilities of packing for abnormal proteins that can cause diseases. Using computational power of multiple systems generates more structures and several search strategies are tested in order to identify the most effective one.

Foldit game is based on the Rosetta platform. The player or groups of players are able to manipulate the amino acid chain to achieve minimum energy conformations, those that achieve the most favorable energy structure win [6].

Another application helps to identify ways in which a number of drugs bind to a receptor of known 3D structure of a HIV component [7].

\section{Methods in medical education that utilizes virtualization}

As a method of education, virtual medicine is used in various ways: either to explore the human body, without interacting with the analyzed structures, or by performing maneuvers on simple or complex structures (up to simulate human organs) to quantify gestures.

Satava describes the features of ideal simulator: high fidelity, capacity to simulate the physicdynamic characteristics of organs, to be interactive and to provide haptic feedback [8]. 
Some examples of the exploration of the human body for anatomical study are: Visible Human Project [9, 10], The Virtual Body [11]; The Virtual Human Embryo [12]; The Visible Human Server [13]. Most of these resources are online atlases of three-dimensional reconstruction images obtained from computerized tomography or MRI. These sites allow interactive navigation and detailed anatomical study of the structures concerned, including relations with other organs.

Three-dimensional reconstruction of intracellular organelles for a better understanding of physiology is another area in which the research was conducted, allowing visualization of how these structures interact [14].

Rapid development of laparoscopic techniques require the implementation of special training and acquisition of special technical skills. Design and modeling of three-dimensional virtual environments that respect the anatomy and intraoperative conditions require close collaboration between dedicated surgeons and engineers in order to develop such platforms. The virtual environment is connected to a console that allows the surgeon to manipulate the virtual instruments through a dedicated joystick. Also some devices use a system for haptic feedback simulation. Simulation is now an integrated part of medical education. These simulators allow the trainee to make mistakes and to learn how to avoid or to solve them, in order to reduce complications in the real procedures.

Of these simulators the best known are: MIST-VR, The LaparoscopyVR [15]; LapMentor [16]; LapSim [17].

MIST-VR system allows the surgeon's training in a virtual space assimilated to a three dimensional cube where the student can perform different moves with a cylinder and balls and the difficulty can be graded in 6 steps. The time to accomplish the tasks as well as the errors are quantified. The system is used to learn simple gestures in laparoscopic surgery, but does not have force-feedback [18].

The surgical simulator LaparoscopyVR (LapVR ${ }^{\mathrm{TM}}$ ) is a virtual reality simulation system that enables learning and updating skills needed in laparoscopic surgery. The LapVR ${ }^{\mathrm{TM}}$ system combines laparoscopic simulation software with haptic hardware, providing a complete unit for the virtual reality training. LapVR ${ }^{\mathrm{TM}}$ was designed to help surgeons in acquiring, maintaining, preserving and assessing movement skills and knowledge necessary to perform various laparoscopic surgery procedures. Clinical studies show that novice surgeons trained by laparoscopic simulation have skills that help them in real procedures. LapVR ${ }^{\mathrm{TM}}$ system allows both individual and team training and provides a basic technical module composed of five skills steps with increasing levels of difficulty: camera navigation, using the hook electrode, cutting, clipping, suturing and knot tying. The system also contains a module for laparoscopic cholecystectomy with 18 variants of cases and three levels of difficulty as well as one of obstetrics and gynecology that allows simulation of laparoscopic operations performed for ectopic pregnancy, tubal occlusion and adnexa's pathology [12].

Multidisciplinary laparoscopic surgical simulator LapMentor ${ }^{\mathrm{TM}}$ provides training opportunities for beginners and experienced surgeons from basic laparoscopic skills to complete laparoscopic operations. LapMentor ${ }^{\mathrm{TM}}$ was designed to meet the needs of training and practice 
of physicians as well as the assessment and management of their instructors. The system is characterized by life-like view of human anatomy and perception of tactile sensations using laparoscopic instruments that mimic reality (haptic system). Application modules provide a relaxing environment outside the operating room to help learning essential skills for a variety of laparoscopic procedures: basic techniques module, suturing module, laparoscopic cholecystectomy module, ventral hernia module, gastric bypass module and gynecology module. The basic techniques module was designed to train surgeons in basic skills in minimally invasive procedures: camera navigation (with $0^{-}$and $30^{\circ}$ laparoscope), handling of usual instruments, manipulating objects, clipping and cutting. Suture techniques module allows surgeons to learn intracorporeal suturing and knot tying, providing a perfect training environment similar to real-life practice. Laparoscopic cholecystectomy module includes virtual patient reconstructions obtained from CT / MRI images of real patients with multiple anatomical variants and thus training surgeons in recognition of anatomical variants and successful resolution of surgical complications. The Ventral hernias module represents the first opportunity to train surgeons with skills necessary to perform laparoscopic hernia repair procedures and inserting prostheses of different shapes and sizes. The gastric bypass module helps surgeons accustomed with basic laparoscopic surgery in learning, performing and perfecting stages of this bariatric procedure in a safe and monitored environment. The gynecological laparoscopic module trains surgeons in learning different methods applied in the treatment of ectopic pregnancy, tubal sterilization and adnexal disease. Videos of all laparoscopic interventions are available before or during simulation. The skills and knowledge acquired during the virtual training increases surgeon's experience, allowing them to cope with intraoperative complications, such as: bleeding from the implantation site of trocars, organ rupture, flooding the abdominal cavity with blood. LapMentor ${ }^{\mathrm{TM}}$ system provides all the elements necessary to validate acquired skills and recorded performances. Detailed information on handling tissue, handling tools, time and movements efficiency are collected during the training session to allow evaluation of the level of skill. Performance indicators are recorded in a file and can be viewed on simulator [19].

LapSim $^{\mathrm{TM}}$ system provides a virtual environment very similar to the real one as well as extremely useful simulations for common practice. The skills acquired through this system are directly transferable to the operating room. The interface is easy to use and practical sessions vary in complexity depending on the chosen level. Courses can be modified by an instructor according to specific needs. Functions can be imported and exported to be transferred between instructors and institutions. Standard modules of this system are: the basics skills, anastomosis and suture, laparoscopic cholecystectomy and one dedicated to gynecology [14].

SINERGIA is a new type of simulator for laparoscopic surgery composed of seven teaching modules: eye-hand coordination, handling the camera, grasping, pulling, cutting, dissection and section. It also can achieve differentiation between levels of student skills. Yet, even this simulator does not have an integrated haptic system [20].

Compared to physical simulators (artificial or animal model), virtual simulators stimulate the learner to perform all duties listed and evaluated by software, it does not require the presence of a permanent trainer to verify the correctness of performing the tasks and evaluates more accurate the gestures of the surgeon. Virtual systems for surgical training can be used for 
assessing the competence of the surgeon. In addition, warm-up concept can bring increased surgical performance. This concept allows the warm-up by making virtual preoperative procedure and later, the surgeon will perform the actual procedure. To improve this concept for preoperative phase in virtual environment, three-dimensional reconstructions created from $\mathrm{CT}$ of the patient to be operated can be used, thus simulating more accurately the patient's own anatomical variations in the virtual environment.

The VERT system is a virtual environment used in the training of radiotherapy physician and provides a platform for acquiring the skills necessary for doctors, nurses and auxiliary staff $[21,22]$.

VirRAD project is an online platform that includes a virtual laboratory where the radiopharmacy community members can experience and learn different aspects of practice [23].

A team from the United States has developed a virtual learning system used for EndoStitch device suture learning and performing laparoscopic nodes. It includes in addition to the virtual environment a working console and a haptic feedback [24].

The usefulness of virtual medicine as a diagnostic method is exemplified through a relatively known example: virtual colonoscopy. This examination allows inspection of virtual colonic lumen obtained using three-dimensional reconstruction based on computed tomography images acquired from a patient. The range of examinations is currently expanded to virtual gastrointestinal exploration, three-dimensional assessment of vascular lumen-especially if coronary obstruction. Also the new geometry of the damaged heart wall after a myocardial infarction could be explored.

For intravenous catheter insertion, CathSim system was developed, that provides the student, besides the virtual environment, with a working console with tactile feedback and a series of simulations of complications that may occur during the real procedure [25].

A large number of simulators were developed for: endoscopic sinus surgery, endoscopic gastrointestinal procedures, bronchoscopy, arthroscopy, cardiac catheterization and ophthalmic surgery [26-29].

By integrating knowledge in the field of acupuncture and three-dimensional reconstruction based on images obtained from computer tomography, Chinese Visible Human was created, which is useful in education and research in Chinese medicine [30].

In dentistry there are 3D models of teeth or of an entire human head simulating the details of anatomy of the teeth or mouth. The trainee could perform drills having a haptic feedback. Also a Virtual Reality Dental Chair system (HapTEL) enables the trainee to test and learn various skills [31].

\section{Methods of diagnostic and treatment using virtualization in medicine}

There are a number of softwares for three-dimensional reconstruction of organs using 3D ultrasound images or 4D CT or MRI images, acquired for a more accurate evaluation of 
diseases. Some software even allows a simulation of the proposed intervention thus achieving preoperative planning.

During remote interventions, the surgeon acts on a virtual environment, the operation itself is performed by a surgical robot. This kind of procedure was performed in September 7, 2001 and was called "Lindbergh operation” during which Professor J. Marescaux located in Strasbourg performed a laparoscopic cholecystectomy on a patient who was in New York (at a distance of approx. $15000 \mathrm{~km}$ ) using a ZEUS robot (Computer Motion), the surgeon's presence being virtual [32].

The utility of this technology is to use the expertise of a specialist at a great distance (i.e. space station) to perform surgery (telemedicine) or to help a less experienced surgeon during interventions with high difficulty (telementoring).

The use of robots in surgery allows more accurate movements, avoiding surgeon's tremor, smaller incisions, decreased blood loss, quicker postoperative recovery, etc.

The new models of modified DaVinci robot uses three-dimensional immersion type image, allowing detailed visualization of the operative field, besides haptic feedback [33].

Virtual reality refers not only to virtualization of visual perception but also to virtualization of tactile perception, the touch feeling being essential in surgery. Haptic systems are designed as a virtual environment for the sense of touch, allowing artificial tactile perception of objects created in the virtual environment or of real objects touched by surgical robot, the sensations being transmitted to the interface with the surgeon.

VerroTouch system is an extension of DaVinci robot proposed by Kuchenbecker et al [34] which makes transfer of vibrations measured by surgical instruments attached to the robotsurgeon interface, allowing haptic sensation to the operator.

Augmented reality facilitates surgical intervention by projecting in the operative field inner images of the organs located in the visual field. These images are obtained by three-dimensional reconstruction from CT or MRI after acquisition and processing. The projected images are adjusted according to the position of organs, instruments and actions of the surgeon in the operating field. For example, large intrahepatic vessels can be projected on the surface of the liver capsule, showing a useful map to the surgeon in order to avoid injuries. This is called also „intraoperative navigation” and it shows the surgeon where he can find important elements and also the anatomic variations.

Navigation systems have been proposed for NOTES (Natural Orifice Transluminal Endoscopic Surgery) surgery $[35,36]$ which reduce intraoperative accidental injuries. It uses threedimensional reconstruction from images captured by CT and data of real-time location of the endoscope. The main problem with augmented reality is identifying common reference points of real images and of those obtained by preoperative three-dimensional reconstruction. Augmented reality is very useful during minimally invasive interventions, where the visual field is diminished and thus the orientation of the surgeon in the operating field is hampered.

The current systems of virtual reality and augmented reality fail to perfectly simulate the interaction between surgical instruments and organs. In the future, the researchers aim to 
improve the interaction between surgeon and interface with the virtual system, getting feedback for surgeons, synchronization of virtual reality with operative field, etc.

Virtual reality can be very useful in bridging the gap between food and social behavior of patients with diabetes and/or obesity and the rules that they should follow. The software allows interaction with virtual environments that simulate both video and tactile sensations and other sensations (smell, taste). Patients can learn new habits such as the amount of food they should ingest, food choices and given encouragement to perform physical activities. Also in people with heart disease or morbid obesity it may stimulate performing progressive physical activity at home, even by increasing motivation.

Another direction in which virtual medicine interferes with spectacular results is the treatment of phobias. The patient is connected to a virtual environment that simulates situations that produce phobic states, everything being controlled and can be interrupted at any time of the simulation [37]. Phobias treated with such reality simulation programs are: post-traumatic stress, public speaking, arachnophobia, agoraphobia, acrophobia, claustrophobia, panic, social phobia, stress secondary vehicle crush accidents etc. [38]. A number of applications have also been developed, dedicated to various phobias or posttraumatic stress syndromes (Virtual Iraq/ Afghanistan, Virtual Airplane, Virtual Nicotine, Virtual Elevator, Hurricane Katrina Virtual, Virtual World Trade Center and Virtual Vietnam) [39]. Virtual Iraq / Afghanistan is used in more than 60 clinics and has demonstrated efficacy in reducing symptoms of posttraumatic stress [40].

STRIVE (Stress Resilience in Virtual Environments) is an application that is used to adapt future soldiers to fight stress. The body reactions to stress are described to the soldier and also he can learn how to cope with this stress [40].

VirtualPatients is an application used for the clinician involved in the learning process and interaction with the patient [40].

For trypanophobia treatment (fear of needles) a system with two lenses was developed which shows images to the patient. It can project different scenarios such as a visit to a health center where a nurse shall pick a blood sample. The whole system also provides feedback on the mental and emotional reactions [41].

To help patients with disabilities, along with dedicated systems which allow the patients to learn how to use wheelchairs in crowded cities, he can also use Second Life type virtual environments, where through an avatar, these patients can interact with others without the limitations encountered in the real world [42].

To avoid animal experiments there were developed digital models of rats, pigs and humans. For example, a virtual pig head [41] and also a model of a physiological rat [43] was developed, in order to use these models in virtual experiments.

Three-dimensional virtual reality combined with haptic feedback is used for motor recovery of patients with stroke sequelae. This technology is currently used in experimental studies, but it might extend in recovery clinics and even at home recovering. There are commercial systems used for related purposes with the ability to develop complex movements of the hand. These 
applications provide an assessment of movements and skills acquired in order to follow-up the evolution of these patients [44, 45].

A new area of research with direct implications in the therapy is currently developing: virtual rehabilitation. Also looming as a subdomain, virtual tele-rehabilitation allows the therapist to interact with the patient through a 3D avatar and guide them during therapy sessions. Haptic systems attached to such systems allow the doctor to examine the patient's muscle tone [46, 47].

Another application is the virtual prototyping technology that combines virtual modeling with rapid prototyping in order to be used in biomedical engineering (eg. for the development of prosthetic or medical devices) [48, 49].

\section{Ethics in virtual reality medicine and telemedicine}

The rapid evolution of virtual reality and telemedicine may result in overcoming limits, more so in this area as yet there are no law or ethics rules. As the interactions between people from different countries become more and more globalized, the implementation of ethical codes should be considered. .

Implementation of very expensive virtual reality and augmented reality systems for use in therapy can create disparities in specialist's presence who will migrate toward the best equipped centers. Also there will be an imbalance in patients addressability, which also would prefer clinics with superior performance $[50,51]$.

Training therapists in centers with the possibility of using virtual reality will also be more efficient and also preoperative simulations leading to superior results. These issues are already visible in Eastern European countries with subfinanced health systems, where both patients and physicians migrate to Western European states.

Funding the development of such performant systems of virtual reality and telemedicine is not currently covered by health systems or government agencies but more often through projects or private sponsorships. These facts may lead to impairing of projects on longterm [52].

As virtual systems will gain autonomy in different degrees, the responsibility of creators and users of these systems will increase. There is currently no legislation to use autonomous robots or virtual systems. There arises the problem of such systems errors and who will be punished for them.

Some cultural and social problems may occur in telemedicine. Because the distance between doctor and patient, the latter can be reduced to a mere "beneficiary" or, worse, "data source" [53].

Differences between health legislations in different countries may lead to situations such as: doctor without a license in a country may provide remote treatment in another country; some illegal procedures (cloning, abortion) in a country can be remotely performed in another country where they are allowed. These differences can lead to self-limitation of experts 
performing remote therapies in countries where laws are not familiar. It is therefore necessary to implement a symmetric legislation.

Storing data in information systems can lead to loss of confidentiality with repercussions on both the patient and the service providers.

Patients are usually informed of these systems by mass-media (stimulated by the industry) and these information is incomplete or insufficient.

\section{Future directions}

Development of virtual reality in medicine aims in the following directions: increasing fidelity in order to reproduce the reality, haptic systems integration in the usual simulators and surgical robots, identifying new standards for evaluating student performance, expanding the use of simulators in the educational field, research and therapy. Virtual reality is likely to become the most common method of learning and training young surgeons and also for senior surgeons to acquire new skills [2].

The researchers also seek solutions to lower the costs of information systems and especially with force feedback haptic devices, but its price makes them prohibitive for general use.

A new direction is predicted, combining virtual reality with holographic projection which would improve the three-dimensional vision.

The combination of autonomous systems with virtual reality will be able to step into the future for autonomous surgical robots, autonomous systems for rehabilitation of people with disabilities, autonomous educational systems and complex software for virtual research.

\section{Conclusion}

The spectrum of applications of virtual reality in medicine widens permanently and it is developing in various unexpected directions. The contribution of IT support in the development of medicine is huge but the possibilities and applications that will shape the future of medicine are still unknown.

Virtual reality finds its applications in various fields and its usefulness becomes more predominant in training, preoperative evaluation and preparation of surgeons. Learning with virtual simulators increases the dexterity of the surgeons and reduces the number and severity of intraoperative complications. Also, in computer-assisted research, virtual reality brings benefits that other methods could not reach. Various models are developed for research purposes at different levels, from macro-models to micro-or even nano-levels allowing researchers to perform "in silico" experiments more rapidly and more cheaply.

The combination of virtual reality with the power of multiple computers as in online communities enhances the speed of research to a level otherwise unachievable at this time. 
The use of virtual reality in the diagnosis and especially in treatment of certain diseases, widens the range of therapeutic procedures, increases operator safety, patient compliance and reduces the duration of applied treatment. Virtual environments used to treat phobias could not be achieved by any other means, leading to a unique mode of treatment in this field.

Using robot technology as well as telemedicine and telementoring systems will increase surgical accuracy and will reduce hospitalization and complications and on the other hand will increase medical expertise in disadvantaged areas or with difficult access (including inaccessible areas for experts: stations space, underwater).

As in all newly developed fields, the virtual reality domain needs some sound rules to be applied during its use, in order to avoid mistakes and/or injuries, especially in the field of diagnostics and therapeutics.

\section{Author details}

Florin Graur ${ }^{1,2^{*}}$

Address all correspondence to: graurf@yahoo.com

1 University of Medicine and Pharmacy "Iuliu Hatieganu" Cluj-Napoca, Romania

2 Regional Institute of Gastroenterology and Hepatology "Octavian Fodor" Cluj-Napoca, Romania

The author has no conflict of interest.

\section{References}

[1] Mann, R.W. The evaluation and simulation of mobility aids for the blind. in Rotterdam Mobility Research Conference. 1965. New York: American Foundation for the Blind.

[2] Custura-Craciun, D., et al., Surgical virtual reality-highlights in developing a high performance surgical haptic device. Chirurgia (Bucur), 2013. 108(6): p. 757-63.

[3] Folding@Home. 2014 [cited 201424 August]; Available from: http://folding.stanford.edu/.

[4] Rosetta@Home. 2014 [cited 201424 August]; Available from: http://boinc.bakerlab.org/ rosetta/.

[5] http://www.worldcommunitygrid.org/. Human Proteome Folding Project 2014 [cited 201428 August]; Available from: http://www.worldcommunitygrid.org/research/ hpf2/overview.do. 
[6] Wikipedia. Rosetta@home. 2014; Available from: http://en.wikipedia.org/w/index.php? title=Rosetta@home\&oldid=616140030.

[7] FightAIDS@Home. [cited 201424 August]; Available from: http://www.worldcommunitygrid.org/research/faah/overview.do.

[8] Satava, R.M., Historical review of surgical simulation--a personal perspective. World J Surg, 2008. 32(2): p. 141-8.

[9] NLM. The Visible Human Project. 2014 [cited 201424 August]; Available from: http:// www.nlm.nih.gov/research/visible/visible_human.html.

[10] NLM. Visible Human. 2014 [cited 201424 August]; Available from: http://www.madsci.org/ lynn/VH/.

[11] The Virtual Body. 2014 [cited 201424 August]; Available from: http://medtropolis.com/virtual-body/.

[12] LSUHSC. Virtual Human Embryo Project. 2011 [cited 201424 August]; Available from: http://virtualhumanembryo.lsuhsc.edu/.

[13] visiblehuman.epfl.ch. Visible Human. 2014 [cited 201424 August]; Available from: http://visiblehuman.epfl.ch/index.php.

[14] Organelle View. 2014 [cited 201424 August]; Available from: http://www.nformationdesign.com/portfolio/portfolio01.php.

[15] caehealthcare.com. LapVR Surgical Simulator. 2014 [cited 201424 August]; Available from: http://www.medsimlab.com/brochuras/lap.pdf.

[16] Simbionix. LAP Mentor. 2014 [cited 201424 August]; Available from: http://simbionix.com/simulators/lap-mentor/.

[17] SurgicalScience. http://www.surgical-science.com. 2014 [cited 201424 August]; Available from: http://www.surgical-science.com.

[18] Tarcoveanu, E., R. Moldovanu, and A. Vasilescu, INNVĂŢĂMÂNTUL VIRTUAL ÎN CHIRURGIA MINIM INVAZIVĂ. Jurnalul de Chirurgie, 2010. 6(3): p. 230-236.

[19] Saunders, N.R., The Celestin tube in the palliation of carcinoma of the oesophagus and cardia. Br J Surg, 1979. 66(6): p. 419-21.

[20] Lamata, P., et al., SINERGIA laparoscopic virtual reality simulator: didactic design and technical development. Comput Methods Programs Biomed, 2007. 85(3): p. 273-83.

[21] www.virtalis.com. VERT: Virtual Environment Radiotherapy Training. 2014 [cited 2014 24 August]; Available from: http://www.virtalis.com/files/articles/flier_whatisvert.pdf.

[22] www.vertual.co.uk. VERT. 2014 [cited 201424 August]; Available from: http:// www.vertual.co.uk/. 
[23] VirRAD. VirRAD. 2014 [cited 201424 August]; Available from: http:// Www.virrad.org/.

[24] Kurenov, S.N., et al., Simulation for training with the Autosuture Endo Stitch device. Surg Innov, 2006. 13(4): p. 283-7.

[25] CathSim Intravenous Simulator. 2014 [cited 201424 August]; Available from: http:// www.pennstatehershey.org/web/simulation/equipment/cathsim.

[26] Bro-Nielsen, M., et al., PreOp endoscopic simulator: a PC-based immersive training system for bronchoscopy. Stud Health Technol Inform, 1999. 62: p. 76-82.

[27] Cotin, S., et al., ICTS, an interventional cardiology training system. Stud Health Technol Inform, 2000. 70: p. 59-65.

[28] Mabrey, J.D., et al., Development of a virtual reality arthroscopic knee simulator. Stud Health Technol Inform, 2000. 70: p. 192-4.

[29] Rudman, D.T., et al., Functional endoscopic sinus surgery training simulator. Laryngoscope, 1998. 108(11 Pt 1): p. 1643-7.

[30] Heng, P.A., et al., Virtual acupuncture human based on chinese visible human dataset. Stud Health Technol Inform, 2006. 119: p. 194-7.

[31] THE Awards: Virtual reality chair helps drill trainee dentists. 2013 [cited 201425 August]; Available from: http://www.reading.ac.uk/sse/news/sse-newsarticle-2013-11-25.aspx.

[32] Marescaux, J., OPERATION LINDBERGH-A World First in TeleSurgery: The Surgical Act Crosses the Atlantic! September 19, 2001: New York-Strasbourg.

[33] Okamura, A.M., Haptic feedback in robot-assisted minimally invasive surgery. Curr Opin Urol, 2009. 19(1): p. 102-7.

[34] Kuchenbecker, K.J., et al. VerroTouch: High-Frequency Acceleration Feedback for Telerobotic Surgery. in EuroHaptics'10 Proceedings of the 2010 International Conference on Haptics: Generating and Perceiving Tangible Sensations. 2010.

[35] Vosburgh, K.G. and R. San Jose Estepar, Natural Orifice Transluminal Endoscopic Surgery (NOTES): an opportunity for augmented reality guidance. Stud Health Technol Inform, 2007. 125: p. 485-90.

[36] Fernandez-Esparrach, G., et al., The role of a computed tomography-based image registered navigation system for natural orifice transluminal endoscopic surgery: a comparative study in a porcine model. Endoscopy, 2010. 42(12): p. 1096-103.

[37] VBI. Virtual Reality Software Programs for Phobias, Substance Use Disorders, Skills Development, PTSD, E Stress/Pain Management. [cited 201424 August]; Available from: http://www.virtuallybetter.com/.

[38] VRMC. Virtual Reality Phobia. [cited 201424 August]; Available from: http:// www.vrphobia.com. 
[39] Virtual Reality Therapy for Phobias. 2014 [cited 201424 August]; Available from: http:// psychiatry.duke.edu/divisions/general-psychiatry/virtual-reality-therapy-phobias.

[40] USC-ICT. Medical Virtual Reality. [cited 201424 August]; Available from: http:// medvr.ict.usc.edu/projects/.

[41] VRS.ORG.UK. Virtual reality and trypanophobia (fear of needles). [cited 201418 Aug]; Available from: http://www.vrs.org.uk/virtual-reality-healthcare/trypanophobia.html.

[42] VRS.ORG.UK. Virtual reality for the disabled. [cited 201418 Aug]; Available from: http://www.vrs.org.uk/virtual-reality-healthcare/disabled.html.

[43] The Virtual Physiological Rat Project. 2014 [cited 201425 August]; Available from: http://virtualrat.org/.

[44] Broeren, J., et al., Assessment and training in a 3-dimensional virtual environment with haptics: a report on 5 cases of motor rehabilitation in the chronic stage after stroke. Neurorehabil Neural Repair, 2007. 21(2): p. 180-9.

[45] Merians, A.S., et al., Sensorimotor training in a virtual reality environment: does it improve functional recovery poststroke? Neurorehabil Neural Repair, 2006. 20(2): p. 252-67.

[46] Sandlund, M., S. McDonough, and C. Hager-Ross, Interactive computer play in rehabilitation of children with sensorimotor disorders: a systematic review. Dev Med Child Neurol, 2009. 51(3): p. 173-9.

[47] ScienceDaily. Pushing boundaries of virtual reality. 2013 [cited 201424 August]; Available from: www.sciencedaily.com/releases/2013/02/130205143336.htm.

[48] Choi, S.H. and H.H. Cheung, Multi-material virtual prototyping for product development and biomedical engineering. Computers in industry archive, 2007. 58(5): p. 438-452.

[49] Clin, J., C.E. Aubin, and H. Labelle, Virtual prototyping of a brace design for the correction of scoliotic deformities. Med Biol Eng Comput, 2007. 45(5): p. 467-73.

[50] Chen, L.C. and J.I. Boufford, Fatal flows--doctors on the move. N Engl J Med, 2005. 353(17): p. 1850-2.

[51] Dickens, B.M. and R.J. Cook, Legal and ethical issues in telemedicine and robotics. Int J Gynaecol Obstet, 2006. 94(1): p. 73-8.

[52] Silverman, R.D., Current legal and ethical concerns in telemedicine and e-medicine. J Telemed Telecare, 2003. 9 Suppl 1: p. S67-9.

[53] Irvine, R., Mediating telemedicine: ethics at a distance. Intern Med J, 2005. 35(1): p. $56-8$. 
